\title{
A Case of Acute Heart Failure due to Immune Checkpoint Blocker Nivolumab
}

\author{
Munish Sharma $^{\mathrm{a}, \mathrm{c}}$, Giselle A. Suero-Abreu ${ }^{\mathrm{b}}$, Bernard Kimª
}

\begin{abstract}
Immune checkpoint inhibitors can be potentially cardiotoxic. It has not been frequently reported in the literature. Cardiomyopathy with these agents can have early onset and may start with non-specific symptoms like fatigue, weakness before presenting with obvious features of acute heart failure. Rapid progression and fulminant course of this disease necessitate high index of clinical suspicion and early diagnosis. High-dose steroids should be instituted early to blunt the immune response against myocardium. Further bigger studies are needed to fully understand the pathogenesis of this condition.
\end{abstract}

Keywords: Immune checkpoint inhibitor; Cardiomyopathy; Acute heart failure

\section{Introduction}

Immune checkpoint inhibitors consist of ipilimumab, an anticytotoxic T-lymphocyte-associated antigen 4 (CTLA-4) antibody, nivolumab and pembrolizumab, which are anti-programmed death-1 (PD-1) antibodies. Nivolumab inhibits PD-1 receptor on $\mathrm{T}$ cells and blocks $\mathrm{PD}-1$ pathway-mediated antitumor immune response inhibition [1]. Apart from malignant melanoma, non-small cell lung cancer (NSCLC) and renal cell carcinoma (RCC), nivolumab has been found to exhibit effective antitumor activity in patients with relapsed or refractory B- and T-cell lymphomas who received pretreatment [2]. Due to aberrant activity of autoreactive $\mathrm{T}$ cells, immune checkpoint inhibitors have been found to be associated with dermatitis, endocrinopathies, colitis, pneumonitis and hepatitis [3]. Cardiotoxicity associated with immune checkpoint inhibitors when used alone or in combination is rare and has been reported only in a few case reports and case series studies $[3,4]$. We present a

Manuscript submitted January 28, 2019, accepted February 15, 2019

aDepartment of Advanced Heart Failure and Pulmonary Hypertension, Hackensack University Medical Center, NJ, USA

${ }^{b}$ Department of Internal Medicine, Rutgers-NJMS, NJ, USA

${ }^{\mathrm{c} C}$ Corresponding Author: Munish Sharma, Department of Advanced Heart Failure and Pulmonary Hypertension, Hackensack University Medical Center, NJ 07601, USA. Email: munishs1@hotmail.com

doi: https://doi.org/10.14740/cr838 case of acute heart failure in a patient started on nivolumab for treatment of angioimmunoblastic T-cell lymphoma (AITL).

\section{Case Report}

A 76-year-old female patient with history of psoriatic arthritis treated with methotrexate and ustekinumab in the past, seizure disorder on leviteracetam and AITL with central nervous system (CNS) involvement was admitted to our hospital on July 19, 2018 due to extreme fatigue and worsening shortness of breath (SOB) over a period of 1 week. Her SOB was new and rapidly worsened to be present even with minimal exertion. She complained of orthopnea but no paroxysmal nocturnal dyspnea. Patient was diagnosed with AITL in January 2018 and was treated with brentuximab vedotin, cyclophosphamide, doxorubicin, prednisone, and etoposide along with intrathecal methotrexate for CNS involvement. She was started on a combination of brentuximab and nivolumab 22 days prior to this hospitalization. On our initial examination in cardiac care unit, her blood pressure was $67 / 48 \mathrm{~mm} \mathrm{Hg}$, heart rate 104 beats per minute (bpm), temperature 97.2 Fahrenheit, respiratory rate 31/ min and saturation $96 \%$ on 1.5 liters of oxygen via nasal cannula. Her total leucocyte count was $11 \times 103$ cells $/ \mathrm{mL}$ (reference range: $4-11 \times 103$ ), hemoglobin $9.7 \mathrm{~g} / \mathrm{dL}$ (reference range: 12 - 15.5), blood urea nitrogen (BUN) $30 \mathrm{mg} / \mathrm{dL}$ (reference range: 5 - 24), creatinine $1.2 \mathrm{mg} / \mathrm{dL}$ (reference range: 0.3 - 1.5), serum sodium $128 \mathrm{mmol} / \mathrm{L}$ (reference range: 134 - 146), potassium 5.3 $\mathrm{mmol} / \mathrm{L}$ (reference range: 3.5 - 5.2), carbon dioxide $\left(\mathrm{CO}_{2}\right) 22$ $\mathrm{mmol} / \mathrm{L}$ (reference range: 24 - 32), troponin I $0.060 \mathrm{ng} / \mathrm{mL}$ (reference range: $0.0-0.030)$ and brain natriuretic peptide (BNP) $892 \mathrm{pg} / \mathrm{mL}$ (reference range: 0 - 100). Electrocardiogram (ECG) showed sinus tachycardia with no ST-T changes (Fig. 1). Chest $\mathrm{X}$-ray revealed interstitial edema and bibasilar pleural parenchymal opacification. Transthoracic echocardiogram (TTE) was significant for severely reduced left ventricular ejection fraction (LVEF) of less than $20 \%$, moderate pericardial effusion, moderate mitral regurgitation (MR), severe tricuspid regurgitation (TR) and mildly dilated right ventricular cavity (Fig. 2a, b). TTE in June 14, 2018 had revealed LVEF of $55 \%$, grade I diastolic dysfunction, mild MR/TR with no pericardial effusion (Table 1, Fig. 3a-c). Computed tomography (CT) chest showed bilateral small pleural effusions and a small new pericardial effusion (Fig. 4). Viral serology from peripheral blood was non-reactive for adenovirus, enterovirus, Coxsackie virus, Ebstein-Barr virus (EBV) and cytomegalovirus (CMV). She was negative for hepatitis $\mathrm{B} /$ hepatitis $\mathrm{C}$ and human immune deficiency virus (HIV) in 


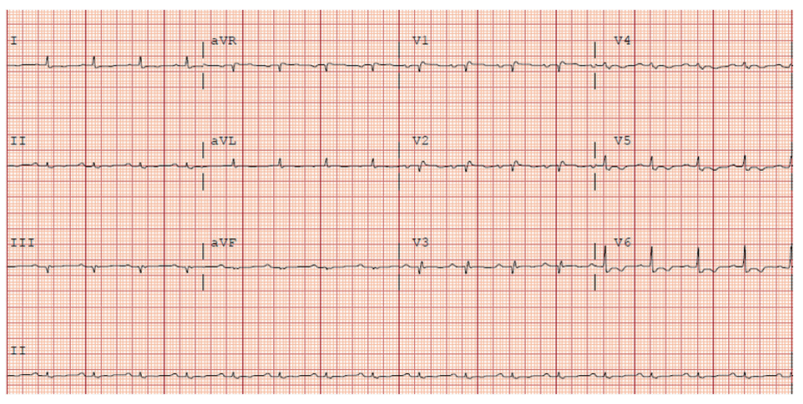

Figure 1. ECG on July 27, 2018: left axis deviation, sinus tachycardia and low voltage.

the recent past. Thyroid-stimulating hormone (TSH) was 2.612 $\mathrm{uIU} / \mathrm{L}$ (reference range: 0.5 - 5.0) and serum protein electrophoresis did not reveal any monoclonal protein peak.

A Swann-Ganz catheter was placed to monitor cardiac hemodynamics. Her cardiac index was $1.5 \mathrm{~L} / \mathrm{min} / \mathrm{m}^{2}$. Patient was started on milrinone drip. Timely mixed venous oxygen saturation was drawn and milrinone was up-titrated to up to 0.6 $\mathrm{mg} / \mathrm{kg} / \mathrm{min}$ to achieve optimal cardiac output. Patient was also on norephinephrine drip to maintain a mean arterial pressure of $65 \mathrm{~mm} \mathrm{Hg}$ or more. She was also treated with $1 \mathrm{mg} / \mathrm{kg}$ of intravenous solumedrol. After 3 days, patient did not show any sign of improvement on inotrope support and underwent implantation of right-sided axillary Impella 5.0 and Impella RP. Repeat TTE on July 28 showed LVEF of less than $20 \%$, severely dilated right ventricle and mild pericardial effusion. Patient continued to remain sedated and ventilated after Impella implantation. An extensive discussion was done between the patient's family members including her power of attorney, primary oncologist and cardiologist. In view of extremely poor prognosis of her widely spread T-cell lymphoma and acute de novo heart failure, family opted to terminally extubate the patient.

\section{Discussion}

Immune checkpoint inhibitors have been found to be promising in inducing antitumor activity in malignancies including

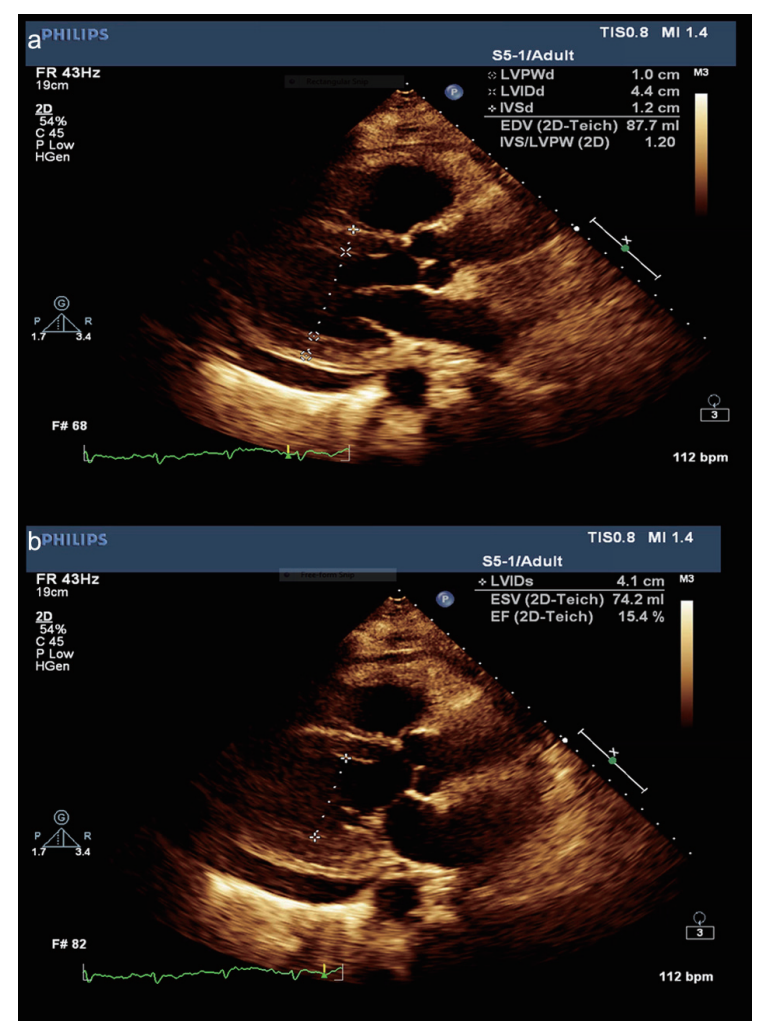

Figure 2. Echocardiogram obtained during hospital admission showing low ejection fraction.

melanoma, NSCLC, RCC and T- and B-cell lymphomas but overall immune-mediated adverse reactions have been found to be as high as $40 \%$ [5]. These agents can have significant cardiac adverse effects that can manifest as autoimmune myocarditis, cardiomyopathy, cardiac fibrosis, heart failure and cardiac arrest [4]. Cardiac toxicity per se has been estimated to occur in less than $1 \%$ patients receiving this immunotherapy. Combination of ipilimumab and nivolumab has been found to produce more severe and frequent cardiotoxicity compared to patients on nivolumab alone [3]. It has been previously reported that two or more organs systems can have simultaneous

Table 1. Two-Dimensional Transthoracic Echogardiogram (TTE) Report on July 21, 2018

\begin{tabular}{|c|c|}
\hline Pericardium & Moderate pericardial effusion \\
\hline Left ventricle & $\begin{array}{l}\text { Severely reduced systolic function, ejection fraction estimated at less than } 20 \% \text {, unable to assess left ventricle diastolic } \\
\text { function (tachycardia), mild asymmetric hypertrophy }\end{array}$ \\
\hline Right atrium & Cavity mildly dilated \\
\hline Inferior vena cava & Dilated $(>2.1 \mathrm{~cm}$ ) with less than $50 \%$ respiratory collapse with an estimated right atrial pressure of $15 \mathrm{~mm} \mathrm{Hg}$ \\
\hline Aortic valve & The aortic valve is sclerotic without reduced excursion, no stenosis, trace regurgitation on color-flow PW Doppler \\
\hline Pulmonic valve & The pulmonic valve was not well visualized, trace regurgitation on color-flow PW Doppler \\
\hline
\end{tabular}

In comparison to the previous study dated June 14,2018 , there is significant change noted (moderate pericardial effusion is seen and LVEF is severely reduced at $<20 \%$ ). 


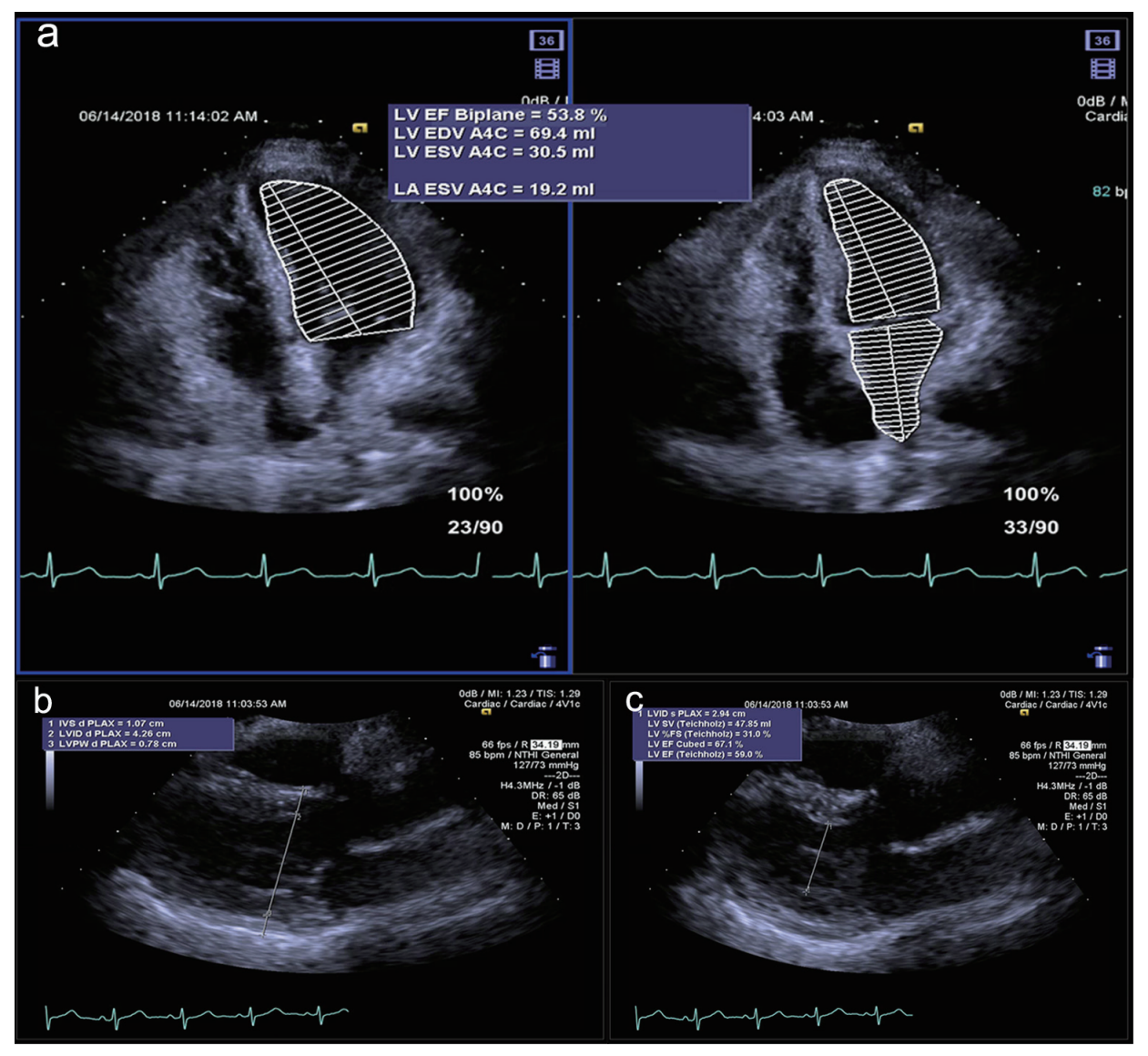

Figure 3. (a) Echocardiogram done on June 14, 2018 showing normal ejection fraction by biplane. (b, c) Echocardiogram done on June 14, 2018.

immune-mediated manifestations with ipilimumab (7\%) and ipilimumab-nivolumab (31\%). Thus special attention needs to be paid for development of cardiotoxicity in those patients who have already experienced an immune-mediated reaction but in absence of predictive biomarkers it is difficult to prevent an impending immune-mediated reaction [6]. The mechanism of adverse events related to immune checkpoint inhibitors has not been fully understood. Studies in mice have shown that deficiency of CTLA-4 induces severe myocarditis due to rampant

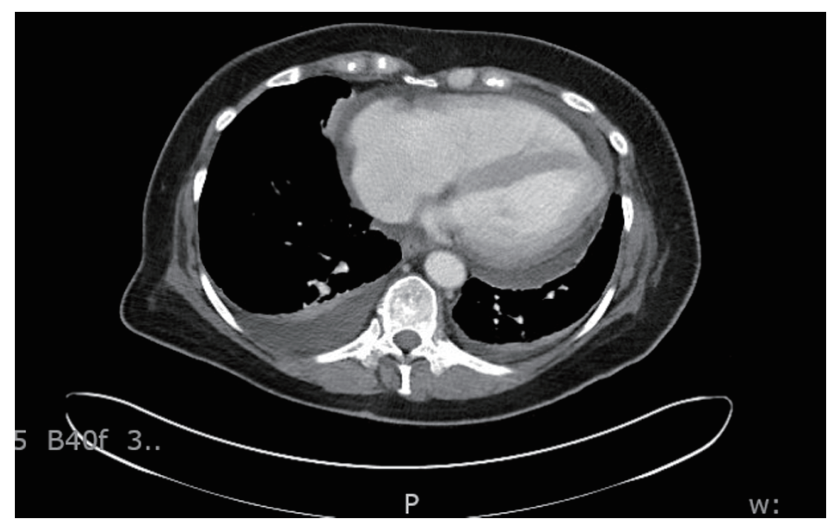

Figure 4. CT chest showing bilateral pleural effusion. lymphocytic infiltration [7]. Since PD-1 is known to be cardioprotective as it inhibits tissue inflammation and myocardial damage, studies have shown that mice with $\mathrm{PD}^{-}{ }^{-} \mathrm{CD}^{+} \mathrm{T}$ cells compared to mice with $\mathrm{PD}^{+/+} \mathrm{CD}^{+} \mathrm{T}$ cells tend to develop severe myocarditis [8]. Spontaneous autoimmune dilated cardiomyopathy can also be seen in mice with PL-1 deficiency [9]. Thus, checkpoint inhibitors likely lower the threshold of activation of T cells against the self-antigen in the heart [4].

In a case series of eight patients, cardiotoxicity occurred within 4 - 22 weeks after initiation of check point inhibitors alone or in combination [4]. In another case series of two patients, fulminant myocarditis occurred within 12 - 15 days of initial therapy. All of these patients were started at $1 \mathrm{mg} / \mathrm{kg}$ of nivolumab alone or in combination $[3,4]$. In our case, patient presented with low output cardiogenic shock within 22 days of initiation of nivolumab $1 \mathrm{mg} / \mathrm{kg}$, which was her only new medication. Endomyocardial biopsy can show features of interstitial inflammation suggested by infiltration of lymphocytes and interstitial fibrosis. Cardiac magnetic resonance imaging (MRI) can reveal enhanced T2 signal suggestive of underlying myocardial edema suggestive of myocarditis. Our patient presented with cardiogenic shock and could not be subjected to endomyocardial biopsy but cardiac MRI could have been contemplated if she had stabilized during the course of treatment. Significant drop in LVEF in echocardiography 
in absence of other identifiable causes can indicate immunemediated cardiotoxicity in patients recently started on immune therapy. Though there is no definite treatment protocol in general, smaller studies have shown improvement in LVEF with intravenous methyl prednisone $1-2 \mathrm{mg} / \mathrm{kg}$ while in the hospital. This can be transitioned to oral prednisone on discharge and tapered over a period of month [4]. There are no bigger studies conducted so far to determine the prognosis of such cases but few case series have shown recovery in around $60 \%$ cases with some permanent decrease in LVEF [3, 4].

\section{Conclusions}

Clinicians must be aware of the potential cardiotoxicity of immune checkpoint inhibitors. It can have early onset and may start with non-specific symptoms like fatigue, weakness before presenting with obvious features of acute heart failure. Rapid progression and fulminant course of this disease necessitate high index of clinical suspicion and early diagnosis. Endomyocardial biopsy can reveal features of myocarditis and fibrosis, but due to its invasive nature, may not be a suitable test in critically ill patients. Careful history, examination and interpretation of basic non-invasive test like echocardiography can give clue to diagnosis. High-dose steroids should be instituted early to blunt the immune response against myocardium. Further bigger studies are needed to fully understand the pathogenesis of this condition so that more specific and effective treatment strategies can be devised.

\section{Conflict of Interest}

Authors declare no conflict of interest.

\section{References}

1. Andorsky DJ, Yamada RE, Said J, Pinkus GS, Betting
DJ, Timmerman JM. Programmed death ligand 1 is expressed by non-hodgkin lymphomas and inhibits the activity of tumor-associated $\mathrm{T}$ cells. Clin Cancer Res. 2011;17(13):4232-4244.

2. Lesokhin AM, Ansell SM, Armand P, Scott EC, Halwani A, Gutierrez M, Millenson MM, et al. Nivolumab in patients with relapsed or refractory hematologic malignancy: preliminary results of a phase Ib study. J Clin Oncol. 2016;34(23):2698-2704.

3. Johnson DB, Balko JM, Compton ML, Chalkias S, Gorham J, Xu Y, Hicks M, et al. Fulminant myocarditis with combination immune checkpoint blockade. N Engl J Med. 2016;375(18):1749-1755.

4. Heinzerling L, Ott PA, Hodi FS, Husain AN, Tajmir-Riahi A, Tawbi H, Pauschinger M, et al. Cardiotoxicity associated with CTLA4 and PD1 blocking immunotherapy. J Immunother Cancer. 2016;4:50.

5. Postow MA, Chesney J, Pavlick AC, Robert C, Grossmann K, McDermott D, Linette GP, et al. Nivolumab and ipilimumab versus ipilimumab in untreated melanoma. N Engl J Med. 2015;372(21):2006-2017.

6. Voskens CJ, Goldinger SM, Loquai C, Robert C, Kaehler $\mathrm{KC}$, Berking $\mathrm{C}$, Bergmann $\mathrm{T}$, et al. The price of tumor control: an analysis of rare side effects of anti-CTLA-4 therapy in metastatic melanoma from the ipilimumab network. PLoS One. 2013;8(1):e53745.

7. Tivol EA, Borriello F, Schweitzer AN, Lynch WP, Bluestone JA, Sharpe AH. Loss of CTLA-4 leads to massive lymphoproliferation and fatal multiorgan tissue destruction, revealing a critical negative regulatory role of CTLA-4. Immunity. 1995;3(5):541-547.

8. Tarrio ML, Grabie N, Bu DX, Sharpe AH, Lichtman AH. PD-1 protects against inflammation and myocyte damage in $\mathrm{T}$ cell-mediated myocarditis. J Immunol. 2012;188(10):4876-4884.

9. Nishimura H, Okazaki T, Tanaka Y, Nakatani K, Hara M, Matsumori A, Sasayama S, et al. Autoimmune dilated cardiomyopathy in PD-1 receptor-deficient mice. Science. 2001;291(5502):319-322. 